Original Research Paper

\title{
Fixed Point Theorems for Mappings Satisfying Weak Nonexpansivity Condition (Weak Contractivity Condition) into (from) Cartesian Products Normed Spaces
}

\author{
Sahar Mohamed Ali Abou Bakr \\ Faculty of Science, Ain Shams University, Cairo, Egypt
}

\section{Article history}

Received: 13-09-2016

Revised: 02-02-2017

Accepted: 10-04-2017

Email: saharm_ali@yahoo.com saharm_ali@sci.asu.edu.eg

\begin{abstract}
This paper suggests new types of weak nonexpansive mappings defined from normed space $X$ into its Cartesian product $X \times X$, studies the main features of the fixed points for those mappings and extends the concept of $(C)$-contractivity condition introduced in some previous research papers. On other side, it introduces new types of contraction mappings with a mixed monotone property; the $\{a, b, c\} \mathrm{M}$-first type and the $\{a, b, c\} \mathrm{M}$-second type contractions, these types are defined from the Cartesian product space $X$ $\times X$ into $X$, where $X$ is a sequentially ordered Banach space, proves the existence of first-anti-second and second-anti-first couple fixed points of such types and generalizes some of the results given before.
\end{abstract}

Keywords: Contraction, Nonexpansive, Quasi Nonexpansiveness Types of Mappings, Mixed Monotone Operators, Mixed Monotone Property, FIXED Points, Couple Fixed Points

MSC: 46, 4705, 47H09, 47H10

\section{Introduction}

Browder (1965) showed that every nonexpansive mapping defined on a bounded, closed and convex subset $C$ of a uniformly convex Banach space $X$ has fixed points. A mapping with assumption weaker than the contractivity is given as:

Definition 1. (Suzuki, 2008)

The self mapping $A$ is called quasi nonexpansive iff:

$$
\|z-A(x)\| \leq\|x-z\| \text { for all } x \in X \text { and } z \in F(A)
$$

where, $F(A)=\{x: A(x)=x\}$.

In 2007, Tomonari Suzuki introduced an intermediate condition between nonexpansiveness and quasi nonexpansiveness on the self mappings $A, A: X \rightarrow X$ and gave some fixed point and convergence theorems for such types of mappings (Suzuki, 2008).

\section{Definition 2 (Suzuki, 2008)}

The self mapping $A$ is said to satisfy the weak $(C)$ condition iff:

$$
\begin{aligned}
& \text { if } x, y \in X \text { and }\left(\frac{1}{2}\right)\|x-A(x)\| \leq\|x-y\| \\
& \text { then }\|A(x)-A(y)\| \leq\|x-y\|
\end{aligned}
$$

Suzuki (2008) showed that any mapping satisfying condition $(C)$ on compact and closed subsets of Banach spaces has fixed point.

In the sequel; the space $X$ is considered to be normed space, the Cartesian product $X \times X$ has the maximum norm $\|(x, y)\|_{\infty}=\max \{\|x\|,\|y\|\}$ and the normed space $X \times X$ with this norm will be denoted by $\left(X \times X,\|.\|_{\infty}\right)$. The mapping $\sigma(x, y, z)$ is defined by:

$$
\sigma(x, y, z)=\max \{\|x-y\|,\|x-z\|\}
$$

This paper is divided into two main sections, the first is on a fixed point theory of mappings into Cartesian product spaces and the second is on a fixed point theory of mappings from Cartesian product spaces, those are as follows:

\section{Mappings Satisfying Weak Nonexpansivity Condition into Cartesian Products of Normed Spaces}

We are going to define a new class of weak nonexpansive type of mappings:

\section{Definition 3}

The mapping $A, A: X \rightarrow X \times X$ is: 
- Satisfying the weak nonexpansivity $(C C)$ condition iff $A$ satisfies the following:

$$
\begin{aligned}
& \text { if } x, y \in X \text { and }\left(\frac{1}{2}\right) \sigma((x-A(x)) \leq\|x-y\| \\
& \text { then }\|A(x)-A(y)\|_{\infty} \leq\|x-y\|
\end{aligned}
$$

- Called quasi nonexpansive iff:

$$
\sigma((z, A(x)) \leq\|x-z\| \text { for all } x \in X \text { and } z \in F(A)
$$

In this study; we showed that the weak nonexpansivity $(C C)$ condition on the mapping $A: X \rightarrow X$ $\times X$ is weaker than the nonexpansivity condition and it is stronger than the quasi nonexpansivity condition, we also discussed basic properties on condition $(C C)$.

\subsection{Preliminaries}

The normed space $X \times X \times X$ with $\sigma$ is characterized by the following:

- $\sigma(x, y, z)=0$ if and only if $x=y=z$

- Homogenous property: If $\lambda$ is a given number, then:

$$
\sigma(\lambda(x, y, z))=|\lambda| \sigma(x, y, z)
$$

- $\quad$ Subadditivity property: For any $x, y, z, r, s$ and $w$ in $X$, we have:

$$
\sigma((x, y, z)+(r, s, w)) \leq \sigma((x, y, z))+\sigma((r, s, w))
$$

If $x, y$ and $z$ are elements in $X$, then we denote $(x,(y$, $z)$ ) to be the triple $(x, y, z)$ and write $x \in(y, z)$ to mean $x$ is one of the two entries of $(y, z)$, equivalently; $x \in\{y, z\}$, that is; $x \in(y, z)$ if and only if $x=y$ or $x=z$.

We have:

\section{Definition 4. (Turkoglu, 2011)}

Let $T$ be a mapping from $X$ into $X \times X, T: X \rightarrow X \times X$. Then the point $x$ is said to be pre fixed point of $T$ if and only if $x \in T(x)$ and $x$ is a fixed point of $T$ iff $T(x)=(x, x)$.

\section{Remark}

The point $\mathrm{x}$ is fixed point of $\mathrm{A}$ iff $\sigma(x, A(x))=0$. In fact; assume $A(x)=(y, z)$, then $\sigma(x, A(x))=0$ if and only if $\sigma(x,(y, z))=0$; equivalently $\sigma(x, y, z)=0$. Therefore, it is equivalent to $x=y=z$ which is equivalent to $A(x)=(x, x)$.

\subsection{Basic Characterization of Mapping Satisfying the $(C C)$ Condition}

The main properties of mapping satisfying the $(C C)$ condition are collected in the next few propositions:

\section{Proposition 1}

Let $A$ be a nonexpansive mapping with a fixed point, $A: X \rightarrow X \times X$. Then $A$ is quasi nonexpansive but the converse is not true.

\section{Proof}

Using the direct definitions of nonexpansive and quasi nonexpansive clears the doubts. To show that the converse is not true in general, we have the following counter example: Let $A$ be the mapping defined from [0, $6]$ into $[0,6] \times[0,6]$ by the following:

$$
A(x)=\left\{\begin{array}{l}
(0,0) \text { if } x \neq 6 \\
(4,4) \text { if } x=6
\end{array}\right.
$$

$A$ has the unique fixed point $z=0$, as $A(0)=(0,0)$, hence $F(A)$ is nonempty set and we have:

- If $x=6$, then $\sigma(z, A(x))=\sigma(0,(4,4))=\max \{\mid 0$ $4|| 0-4 \mid,\}=4<6=|6|=\|x\|=\|x-0\|=\|x-z\|$

- If $x \neq 6$, then $\sigma(z, A(x))=\sigma(0,(0,0))=\max \{|0-0|, \mid 0$ $0 \mid\}=0 \leq\|x\|=\|x-0\|=\|x-z\|$

These showed that $A$ is quasi nonexpansive mapping, it is not nonexpansive because it is not continuous.

\section{Proposition 2}

Let $A$ be a nonexpansive mapping, $A: X \rightarrow X \times X$. Then $A$ is satisfying the weak nonexpansivity $(C C)$ condition, the converse is not necessarily true in general.

\section{Proof}

Let $A$ be nonexpansive. Then:

$$
\|A(x)-A(y)\|_{\infty} \leq\|x-y\| \text { for every } x, y \in X
$$

no restrictions have been taken on $x, y$ in $X$, hence it is true in particular for every $x, y$ in $X$ those satisfying $\left[\frac{1}{2}\right]$ $\sigma(x, A(x)) \leq\|x-y\|$. Hence in particular if $x$ and $y$ are elements in $X$ and $\left[\frac{1}{2}\right] \sigma(x, A(x)) \leq\|x-y\|$, then $\| A(x)$ $A(y)\left\|_{\infty} \leq\right\| x-y \|$. To show that there is mapping satisfying condition $(C C)$ which is not nonexpansive, we consider counter example: Let $A$ be the mapping de fined from [0, $7]$ into $[0,7] \times[0,7]$ by the following:

$$
A(x)=\left\{\begin{array}{l}
\left(0, \frac{x}{7}\right) \text { if } x \neq 7 \\
(1,1) \text { if } x=7
\end{array}\right.
$$

$A$ has the unique fixed point $z=0$. In fact; if $x=7$, then 7 is not one of the two entries of the ordered pair (1, 
1), this shows that 7 is not a pre fixed or fixed of $A$, while if $x$ does not equal 7, $x$ is one of the two entries of $A(x)=\left(0, \frac{x}{7}\right)$, then $x \in\left\{0, \frac{x}{7}\right\}$, thus either $x=0$ or $x=\frac{x}{7}$, in both we have $x=0$. The mapping $A$ is not nonexpansive because for $x=6.5$ and $y=7$, we have $A(6.5)=\left(0, \frac{65}{70}\right)$, $A(7)=(1,1)$, hence $\|A(x)-A(y)\|_{\infty}=\|\left(0-1,(65 / 70-1) \|_{\infty}=1\right.$ and $\| x-y||=|6.5-7|=0.5$. Consequently:

$$
\|A(x)-A(y)\|_{\infty}>\|x-y\|
$$

On the other side we show that $A$ satisfies the weak nonexpansivity condition $(C C)$; for that we study the possibilities of $x$ and $y$ in $X$ those satisfying condition $(C C)$. We have the following cases:

- If $x=y=7$, then:

$$
\begin{aligned}
& {\left[\frac{1}{2}\right] \sigma(x, A(x))=\left(\frac{1}{2}\right) \sigma(7,1,1)} \\
& =\left[\frac{1}{2}\right] \max \{\|7-1\|,\|7-1\|\}=3 \\
& \nless|7-7|=0=\|x-y\|
\end{aligned}
$$

This case is out of discussion.

- If $x=7$ and $y \in[1,4]$, then:

$$
\begin{aligned}
& {\left[\frac{1}{2}\right] \sigma(x, A(x))=\left(\frac{1}{2}\right) \sigma(7,1,1)} \\
& =\left[\frac{1}{2}\right] \max \{\|7-1\|,\|7-1\|\}=3 \\
& \text { and }|7-y|=\|x-y\| \geq 3
\end{aligned}
$$

Hence:

$$
\left[\frac{1}{2}\right] \sigma(x, A(x)) \leq\|x-y\|
$$

for such a $y$, we see that:

$$
\begin{aligned}
& \|A(x)-A(y)\|_{\infty}=\left\|(1,1)-\left(0, \frac{y}{7}\right)\right\|_{\infty}=\left\|\left(1,1-\frac{y}{7}\right)\right\|_{\infty} 1, \\
& \text { and } 3 \leq|x, y|=|7-y|<6
\end{aligned}
$$

Hence:

$$
\|A(x)-A(y)\|_{\infty}\|x-y\|, \text { for } x=7 \text { and each } y \in[1,4]
$$

- If $x=7$ and $y \in(4,7]$, then:

$$
\begin{aligned}
& {\left[\frac{1}{2}\right] \sigma(x, A(x))=\left[\frac{1}{2}\right] \sigma(7,1,1)} \\
& =\left[\frac{1}{7}\right] \max \{\|7-1\|,\|7-1\|\}=3 \\
& \text { and } 0<|7-y|=\|x-y\|<3
\end{aligned}
$$

Hence case out of discussion.

- If $x \in\left[0, \frac{14}{3}\right)$ and $y=7$, then:

$$
\begin{aligned}
& {\left[\frac{1}{2}\right] \sigma(x, A(x))=\left[\frac{1}{2}\right] \sigma\left(x, 0, \frac{x}{7}\right)} \\
& =\left[\frac{1}{2}\right] \max \left\{\|x-0\|,\left\|x-\frac{x}{7}\right\|\right\}=\frac{x}{2} \\
& \text { and } \frac{x}{2}<\frac{14}{6}=\frac{7}{3} \leq|x-7|=\|x-y\|=7-x<7
\end{aligned}
$$

Hence $\left[\frac{1}{2}\right] \sigma(x, A(x)) \leq\|x-y\|$, for such $x$ and $y$, we see that:

$$
\begin{aligned}
& \|A(x)-A(y)\|_{\infty}=\left\|\left(0, \frac{x}{7}\right)-(1,1)\right\|_{\infty}=\left\|\left(1,1-\frac{x}{7}\right)\right\|_{\infty}=1, \\
& \text { and } 1<\frac{7}{3}=\frac{21}{3}-\frac{14}{3}=7-\frac{14}{3}<|x-y|=7-x \mid
\end{aligned}
$$

Hence:

$$
\|A(x)-A(y)\|_{\infty}<\|x-y\|, \text { for each } x \in\left[0, \frac{14}{3}\right) \text { and } y=7
$$

- If $x \in\left(\frac{14}{3}, 7\right]$ and $y=7$, then:

$$
\begin{aligned}
& {\left[\frac{1}{2}\right] \sigma(x, A(x))=\left[\frac{1}{2}\right] \sigma\left(x, 0, \frac{x}{7}\right)} \\
& =\left[\frac{1}{2}\right] \max \left\{\|x-0\|,\left\|x-\frac{x}{7}\right\|\right\}=\frac{x}{2} \\
& \text { and } \frac{x}{2}<\frac{28}{6}=\frac{14}{3}>|x-7|=\|x-y\|
\end{aligned}
$$

Hence case out of discussion. More clearly, for $x=$ 6.5 and $y=7$ we have:

$$
\begin{aligned}
& {\left[\frac{1}{2}\right] \sigma(x, A(x))=\left[\frac{1}{2}\right] \sigma\left(x, 0, \frac{x}{7}\right)} \\
& =\left[\frac{1}{2}\right] \max \left\{\|x-0\|,\left\|x-\frac{x}{7}\right\|\right\}=\frac{13}{4} \\
& \text { and } \frac{x}{2}=\frac{13}{4}>|6.5-7|=\frac{1}{2}=\|x-y\|
\end{aligned}
$$


- If $x \neq 7$ and $\neq 7$, then $A$ is nonexpansive from $(0,7)$ into $(0,7) \times(0,7)$ :

$$
\begin{aligned}
& \|A(x)-A(y)\|_{\infty}=\left\|\left(0, \frac{x}{7}\right)-\left(0, \frac{y}{7}\right)\right\|_{\infty}=\|\left(0, \frac{1}{7}(x-y) \|_{\infty}\right. \\
& -\frac{1}{7}\|x-y\|, \text { for } x, y \in(0,7)
\end{aligned}
$$

That is why no need to study the $(C C)$ condition because it is noexpansive in this domain. Hence $T$ is a mapping satisfying the weak nonxpansivity $(C C)$ condition.

\section{Proposition 3}

If $A$ is satisfying the (CC) condition and owns a fixed point, then it is quasi nonexpansive, the converse may not be true in general.

\section{Proof}

Let $z$ be a fixed point of $A, A(z)=(z, z)$ and $x$ be an arbitrarily element of $X, x \in X$. Since:

$$
\left[\frac{1}{2}\right] \sigma(z, A(z))=\sigma(z, z, z)=0 \leq\|z-x\|
$$

The weak nonexpansivity $(C C)$ condition implies:

$$
\|A(x)-A(z)\|_{\infty} \leq\|z-x\|
$$

Hence; if $A(x)=\left(x_{1}, x_{2}\right)$, then we have:

$$
\begin{aligned}
& \|A(z)-A(y)\|_{\infty}=\left\|(z, z)-\left(x_{1}, x_{2}\right)\right\|_{\infty} \leq\|z-x\|, \\
& \max \left\{\left\|z-x_{1}\right\|,\left\|z-x_{2}\right\|\right\} \leq\|z-x\|, \\
& \sigma\left(z, x_{1}, x_{2}\right) \leq\|z-x\|, \\
& \sigma(z, A(x)) \leq\|z-x\|
\end{aligned}
$$

To show that there is a quasi nonexpansive mapping which does not satisfy the $(C C)$ condition, we consider the mapping $A$ that is defined from $[0,5]$ into $[0,5] \times[0$, 5] by the following:

$$
A(x)=\left\{\begin{array}{l}
(0,0) \text { if } x \neq 5 \\
(4,4) \text { if } x=5
\end{array}\right.
$$

It has the unique fixed point $z=0$, as $A(0)=(0,0)$, $F(T)$ is nonempty set and $A$ is quasi nonexpansive mapping. However, since for $x=5$ and $y=4$, we have:

$$
\begin{aligned}
& {\left[\frac{1}{2}\right] \sigma(x, A(x))=\left[\frac{1}{2}\right] \sigma(5, A(5))=\left[\frac{1}{2}\right] \sigma(5,4,4)} \\
& =\left[\frac{1}{2}\right] \max \{|5-4|,|5-4|\}=1=|5-4|
\end{aligned}
$$

and:

$$
\begin{aligned}
& \|A(x)-A(y)\|_{\infty}=\|(4,4)-(0,0)\|_{\infty}=4 \\
& >1=|5-4|
\end{aligned}
$$

Hence; $A$ is not satisfying the weak nonexpansivity $(C C)$ condition.

We have the following:

\section{Definition 5}

If $A$ is a mapping, $A: X \rightarrow X \times X$, then the two components of $A$ denoted by $A_{1}$ and $A_{2}$ are the operators defined by $A_{1}(x)=$ the first entry of $A(x)$ and $A_{2}(x)=$ the second entry of $A(x)$ respectively:

$$
A(x)=\left(A_{1}(x), A_{2}(x)\right)
$$

We also have the following propositions:

\section{Proposition 4}

Let $A$ be a mapping from $X$ into $\left(X \times X,\|.\|_{\infty}\right)$. Then $A$ is satisfying the weak nonexpansivity $(C C)$ condition if and only if both $A_{1}$ and $A_{2}$ are satisfying the weak nonexpansivity $(C)$ condition.

\section{Proof}

Let $A$ satisfy the weak nonexpansivity $(C C)$ condition, we are going to show that the two operators $A_{1}$ and $A_{2}$ are satisfying the weak nonexpansivity $(C)$ condition. Suppose that $\left[\frac{1}{2}\right]\left\|x-A_{1}(x)\right\| \leq\|x-y\|$ and $\left[\frac{1}{2}\right]\left\|x-A_{2}(x)\right\| \leq\|x-y\|$. Then:

$$
\max \left\{\left[\frac{1}{2}\right]\left\|x-A_{1}(x)\right\|,\left[\frac{1}{2}\right]\left\|x-A_{2}(x)\right\|\right\} \leq\|x-y\|
$$

or:

$$
\left[\frac{1}{2}\right] \max \left\{\left\|x-A_{1}(x)\right\|,\left\|x-A_{2}(x)\right\|\right\} \leq\|x-y\|
$$

or:

$$
\left[\frac{1}{2}\right] \sigma\left(x, A_{1}(x), A_{2}(x)\right) \leq\|x-y\| .
$$

Hence:

$$
\left[\frac{1}{2}\right] \sigma(x, A(x)) \leq\|x-y\|
$$

Using the weak nonexpansivity (CC) condition of $A$, we see that:

$\|A(x)-A(y)\|_{\infty} \leq\|x-y\|$ 
Thus:

$$
\begin{aligned}
& \left\|A_{1}(x)-A_{1}(y)\right\|_{\infty} \\
& \leq \max \left\{\left\|A_{1}(y)-A_{1}(x)\right\|,\left\|A_{2}(y)-A_{2}(x)\right\|\right\} \\
& =\left\|\left(A_{1}(y)-A_{1}(x), A_{2}(y)-A_{2}(x)\right)\right\|_{\infty} \\
& =\left\|\left(A_{1}(x), A_{2}(x)\right)-\left(A_{1}(y), A_{2}(y)\right)\right\|_{\infty} \\
& =\|A(x)-A(y)\|_{\infty}
\end{aligned}
$$

Using (1.1) and (1.2) shows that $\left\|A_{1}(y)-A_{1}(x)\right\| \leq\|x-y\|$ this proves that $A_{1}$ satisfies the weak nonexpansivity $(C)$ condition. Similarly, since:

$$
\begin{aligned}
& \left\|A_{2}(x)-A_{2}(y)\right\| \\
& \leq \max \left\{\left\|A_{1}(y)-A_{1}(x)\right\|,\left\|A_{2}(y)-A_{2}(x)\right\|\right\} \\
& =\|A(x)-A(y)\|_{\infty}
\end{aligned}
$$

Using (1.1) and (1.3) gives $\left\|A_{2}(y)-A_{2}(x)\right\| \leq\|x-y\|$ which proves that $A_{2}$ satisfies the weak nonexpansivity (C) condition. Conversely, suppose that both $A_{1}$ and $A_{2}$ are satisfying the weak nonexpansivity $(C)$ condition and $\left[\frac{1}{2}\right] \sigma(x, A(x)) \leq\|x-y\|$, since:

$$
\begin{aligned}
& {\left[\frac{1}{2}\right]\left\|x-A_{1}(x)\right\|} \\
& \leq\left[\frac{1}{2}\right] \max \left\{\left\|x-A_{1}(x)\right\|,\left\|x-A_{2}(x)\right\|\right\}=\left[\frac{1}{2}\right] \sigma(x, A(x))
\end{aligned}
$$

and:

$$
\begin{aligned}
& {\left[\frac{1}{2}\right]\left\|x-A_{2}(x)\right\|} \\
& \leq\left[\frac{1}{2}\right] \max \left\{\left\|x-A_{1}(x)\right\|,\left\|x-A_{2}(x)\right\|\right\}=\left[\frac{1}{2}\right] \sigma(x, A(x))
\end{aligned}
$$

We get $\left[\frac{1}{2}\right]\left\|x-A_{1}(x)\right\| \leq \sigma(x, A(x))$ and $\left[\frac{1}{2}\right]\left\|x-A_{2}(x)\right\|$ $\leq \sigma(x, A(x))$. These imply $\left\|A_{1}(x)-A_{1}(y)\right\| \leq\|x-y\|$ and $\left\|A_{2}(x)-A_{2}(y)\right\| \leq\|x-y\|$. Therefore:

$\max \left\{\left\|A_{1}(x)-A_{1}(y)\right\|,\left\|A_{2}(x)-A_{2}(y)\right\|\right\} \leq\|x-y\|$

Now:

$$
\begin{aligned}
& \|A(x)-A(y)\|_{\infty}=\left\|\left(A_{1}(x), A_{2}(x)\right)-\left(A_{1}(y), A_{2}(y)\right)\right\|_{\infty} \\
& =\left\|\left(A_{1}(x)-A_{1}(y), A_{2}(x)-A_{2}(y)\right)\right\|_{\infty} \\
& =\max \left\{\left\|A_{1}(x)-A_{1}(y)\right\|,\left\|A_{2}(x)-A_{2}(y)\right\|\right\}
\end{aligned}
$$

Using (1.4) and (1.5) gives $\|A(y)-A(x)\|_{\infty} \leq\|x-y\|$ which proves that $A$ satisfies the weak nonexpansivity $(C C)$ condition.

\section{Proposition 5}

The element $x \in X$ is common fixed point of the two components $A_{1}$ and $A_{2}$ iff it is fixed point of $A$.

\section{Proof}

If $x$ is fixed of both $A 1$ and $A_{2}$, then $A_{1}(x)=x$ and $A_{2}(x)$ $=x$, therefore $\left(A_{1}(x), A_{2}(x)\right)=(x, x)$, hence $A(x)=(x, x)$. Now; if $x$ is a fixed of $A$, then $A(x)=(x, x)$, thus $\left(A_{1}(x)\right.$, $\left.A_{2}(x)\right)=(x, x)$, consequently $A_{1}(x)=x$ and $A_{2}(x)=x$.

\section{Proposition 6}

The element $x$ is prefixed of $A$ iff it is fixed of either $A_{1}$ or $A_{2}$.

\section{Proof}

Suppose that $x$ is a prefixed of $A$. Hence $x \in A(x)$, $x \in\left(A_{1}(x), A_{2}(x)\right)$, that is; either $x=A_{1}(x)$ or $x=A_{2}(x)$ and vice versa.

Finally; we have:

\section{Corollary 1}

Suppose that $K$ is a compact convex subset of a Banach space $X, A$ is a mapping $A: K \rightarrow K \times K$. If $A$ satisfies the weak (CC) condition, then $A$ has at least one prefixed point.

Proof

Let $A$ satisfy the weak $(C C)$ from $K$ into $K \times K, A$ : $K \rightarrow K \times K$. Using Proposition (4) shows that both $A_{1}$ and $A_{2}$ are satisfying the weak $(C)$ condition as mappings from $K$ into $K$, this implies the existence of two elements $x$ and $y$ in $K$ such that $A_{1}(x)=x$ and $A_{2}(y)=y$. Now; $A(x)$ $=\left(A_{1}(x), A_{2}(x)\right)=\left(x, A_{2}(y)\right)$ and $A(y)=\left(A_{1}(y), A_{2}(y)\right)=$ $\left(A_{1}(y), y\right)$ proves that $x \in A(x)$ and $y \in A(y)$. That is; both $x$ and $y$ are prefixed points of $A$.

For mappings defined on Cartesian products spaces, we have the following:

\section{Fixed Point Theorems for Mappings Satisfying Weak Contraction Condition from the Cartesian product of Normed Spaces}

In this section, we introduce new type of contraction mappings, these types will be denoted by $\{a, b, c\} \mathrm{M}$ first and $\{a, b, c\}$ M-second, the types are defined from $X \times X$ into $X$, where $X$ is a sequentially ordered Banach space and having mixed monotone property and then we prove the existence of pair, first-anti-second and second-anti-first couple fixed points of such mappings which is in fact generalization of fixed point theorem given in (Ali, 2013). 


\subsection{Introduction and Preliminaries}

Definition 6. (Jain et al., 2014)

A point $x_{0} \in X$ is a pair fixed point of the mapping $T$ from $X \times X$ into $X$ iff $T\left(\left(x_{0}, x_{0}\right)\right)=x_{0}$.

Gnana Bhaskar and Lakshmikanthan (2006) considered a mixed monotone mapping in a Banach space endowed with partial order, using a weak contractivity type assumption to prove the following:

\section{Theorem 1}

Suppose that $X$ is a sequentially lower-upper ordered Banach space, $A: X \times X \rightarrow X$ a mapping having the firstanti-second and first-second mixed monotone properties and satisfy the following condition:

$$
\|A(x, y)-A(u, v)\| \leq \frac{k}{2}[\|x-u\|+\|y-v\|]
$$

for all $x, y, u$ and $v$ in $X$ with $x \geq u$ and $y \leq v$, where $0 \leq k<$ 1. Then $A$ has a first-anti-second couple fixed point in $X$.

Ali (2013) generalized the T. G. Bhaskar and V. Lakshmikantham's result to the mixed monotone mapping on a closed convex and weakly Cauchy subset of a sequentially ordered normed space those satisfying the condition:

$$
\begin{aligned}
& \|T(x, y)-T(u, v)\| \\
& \leq a \max \{\|x-u\|, \| y-v\}+b\|T(x, y)-x\| \\
& +c \max \{\|T(u, v)-u\|,\|T(v, u)-v\|\}
\end{aligned}
$$

for all $x, y, u$ and $v$ in $C$ with $x \geq u$ and $y \leq v$, where $0 \leq$ $a, b, c<1$ and $a+b+c<1$.

We have the following definitions:

\section{Definition 7. (Ali, 2013)}

Let $(X, \leq)$ be a partially ordered set and $T$ be a mapping from $X \times X$ into $X$. Then:

- $T$ is having the first-second mixed monotone property iff $T(x, y)$ is monotone non decreasing in $x$ and is monotone non increasing in $y$, that is; for any $x, y \in X$ :

$$
\text { if } x_{1}, x_{2} \in X \text { and } x_{1} \leq x_{2} \text {, then } T\left(x_{1}, y\right) \leq T\left(x_{2}, y\right)
$$

and:

$$
\text { if } y_{1}, y_{2} \in X \text { and } y_{1} \leq y_{2} \text {, then } T\left(x, y_{1}\right) \geq T\left(x, y_{2}\right)
$$

- $T$ is having the second-first mixed monotone property iff $T(x, y)$ is monotone non increasing in $x$ and is monotone non decreasing in $y$, that is, for any $x, y \in X$ :

$$
\text { if } x_{1}, x_{2} \in X \text { and } x_{1} \leq x_{2} \text {, then } T\left(x_{1}, y\right) \geq T\left(x_{2}, y\right)
$$

and:

$$
\text { if } y_{1}, y_{2} \in X \text { and } y_{1} \leq y_{2} \text {, then } T\left(x, y_{1}\right) \leq T\left(x, y_{2}\right)
$$

- The element $(x, y)$ is first-anti-second couple fixed point of the mapping $T$ iff $T(x, y)=x$ and $T(y, x)=y$

- The element $(x, y)$ is second-anti-first couple fixed point of the mapping $T$ iff $T(x, y)=y$ and $T(y, x)=x$

\section{Definition 8}

Let $(X, \leq,\|\|$.$) be a partially ordered normed space.$ Then $X$ is sequentially lower-upper ordered space if it fulfills the following:

- If $\left\{x_{n}\right\}_{n \in N}$ is a non decreasing sequence in $X$ which converges strongly to $x$, then $x_{n} \leq x$ for all $n \in N$

- If $\left\{y_{n}\right\}_{n \in N}$ is a non increasing sequence in $X$ which converges strongly to $y$, then $y_{n} \geq y$ for all $n \in N$

\section{Definition 9}

Let $(X, \leq,\|\|$.$) be a partially ordered normed space and$ $T$ be a mapping from $X \times X$ into $X$. Then:

- $\quad T$ is $\{a, b, c\}$ first-type contraction mapping on $X$ iff there are real numbers $0 \leq a, b, c<1$ with $a+b+c$ $<1$ and:

$$
\begin{aligned}
& \|T(x, y)-T(u, v)\| \\
& \leq a \max \{\|x-u\|, \| y-v\}+b\|T(x, y)-x\| \\
& +c \max \{\|T(u, v)-u\|,\|T(v, u)-v\|\}
\end{aligned}
$$

for all $x, y, u$ and $v$ in $X$ with $x \geq u$ and $y \leq v$.

- $\quad T$ is $\{a, b, c\}$ second-type contraction on $X$ iff there are real numbers $0 \leq a, b, c<1$ with $a+b+c<1$ and:

$$
\begin{aligned}
& \|T(x, y)-T(u, v)\| \\
& \leq a \max \{\|x-u\|, \| y-v\}+b\|T(x, y)-x\| \\
& +c \max \{\|T(u, v)-u\|,\|T(v, u)-v\|\}
\end{aligned}
$$

for all $x, y, u$ and $v$ in $X$ with $u \geq x$ and $v \leq y$.

- A couple $\left(x_{0}, y_{0}\right)$ is lower-anti-upper coupled of $T$ iff:

$$
x_{0} \leq T\left(x_{0}, y_{0}\right) \text { and } y_{0} \geq T\left(y_{0}, x_{0}\right)
$$


- $\quad T$ is said to have a lower-upper property if and only if $T$ has at least one lower-anti-upper coupled point

- A couple $\left(x_{0}, y_{0}\right)$ is said to be upper-anti-lower coupled of $T$ iff:

$$
T\left(x_{0}, y_{0}\right) \leq x_{0} \text { and } T\left(y_{0}, x_{0}\right) \geq y_{0}
$$

- $\quad T$ is said to have an upper-lower property if and only if $T$ has at least one upper-anti-lower coupled point

Now; we introduce the following new defined types of contraction mappings:

\section{Definition 10:}

- $\quad T$ is $\{a, b, c\}$ M first-type contraction mapping on $X$ iff there exist real numbers $0 \leq a, b, c<1$ with $b<$ $\frac{1}{2}, a+2 b+c<1$ and:

$$
\begin{aligned}
& \|T(x, y)-T(u, v)\| \leq a \max \{\|x-u\|,\|y-v\|\} \\
& +b \max \{\|T(x, y)-x\|,\|T(y, x)-y\|\} \\
& +c \max \{\|T(u, v)-u\|,\|T(v, u)-v\|\}
\end{aligned}
$$

for all $x, y, u$ and $v$ in $X$ with $x \geq u$ and $y \leq v$.

- $\quad T$ is $\{a, b, c\}$ Msecond-type contraction on $X$ if and only if there are real numbers $0 \leq a, b, c<1$ with $b$ $<\frac{1}{2}, a+2 b+c<1$ and:

$$
\begin{aligned}
& \|T(x, y)-T(u, v)\| \leq a \max \{\|x-u\|,\|y-v\|\} \\
& +b \max \{\|T(x, y)-x\|,\|T(y, x)-y\|\} \\
& +c \max \{\|T(u, v)-u\|,\|T(v, u)-v\|\}
\end{aligned}
$$

for all $x, y, u$ and $v$ in $X$ with $u \geq x$ and $v \leq y$

\subsection{Main Results}

We have the following:

\section{Lemma 1}

Suppose that $X$ is a partially ordered normed space, $T: X \times X \rightarrow X$ is an $\{a, b, c\} M$ first-type contraction mapping satisfying (2.1) and (2.2) and $\left(x_{0}, y_{0}\right)$ is lower-anti-upper couple of $T$. Define the sequences $\left\{x_{n}\right\}_{n \in N}$ and $\left\{y_{n}\right\}_{n \in N}$ iteratively by:

$$
\left\{x_{n}\right\}_{n \in N},\left\{y_{n}\right\}_{n \in N}\left\{\begin{array}{l}
x_{1}=T\left(x_{0}, y_{0}\right), y_{1}=T\left(y_{0}, x_{0}\right) \\
x_{2}=T\left(x_{1}, y_{1}\right), y_{2}=T\left(y_{1}, x_{1}\right) \\
\cdots \\
x_{n}=T\left(x_{n-1}, y_{n-1}\right), y_{n}=T\left(y_{n-1}, x_{n-1}\right)
\end{array}\right.
$$

Then the following hold:

(1) If we take $K=\max \left\{\left\|T\left(y_{0}, x_{0}\right)-y_{0}\right\|,\left\|T\left(x_{0}, y_{0}\right)-x_{0}\right\|\right\}$, then:

$$
\begin{aligned}
& \left\|x_{n+1}-x_{n}\right\| \leq\left[\frac{a+c}{1-2 b}\right]^{n} K \\
& \left\|y_{n+1}-y_{n}\right\| \leq\left[\frac{a+c}{1-2 b}\right]^{n} K
\end{aligned}
$$

(2) Both of $\left\{x_{n}\right\}_{n \in N}$ and $\left\{y_{n}\right\}_{n \in N}$ are Cauchy sequences.

\section{Proof}

Using the properties (2.1) and (2.2) of the mixed monotone mapping $T$ gives:

$$
x_{0} \leq x_{1}, y_{1} \leq y_{0}
$$

Consequently:

$$
T\left(x_{0}, y_{0}\right) \leq T\left(x_{1}, y_{0}\right) \leq T\left(x_{1}, y_{1}\right)=x_{2}
$$

In general, one has:

$$
x_{0} \leq x_{1} \leq \ldots, x_{n}, \ldots \text { and } y_{0} \geq y_{1} \geq, \ldots, y_{n}, \ldots
$$

We have the following:

$$
\begin{aligned}
& \left\|x_{n}-x_{n-1}\right\|=\left\|T\left(x_{n-1}, y_{n-1}\right)-T\left(x_{n-2}, y_{n-2}\right)\right\| \\
& \leq a \max \left\{\left\|x_{n-1}-x_{n-2}\right\|,\left\|y_{n-1}-y_{n-2}\right\|\right\} \\
& +b \max \left\{x_{n}-x_{n-1}\|,\| y_{n}-y_{n-1} \|\right\} \\
& +c \max \left\{x_{n-1}-x_{n-2}\|,\| y_{n-1}-y_{n-2}\right\} \\
& \leq(a+c) \max \left\{\left\|x_{n-1}-x_{n-2}\right\|, \| y_{n-1}-y_{n-2}\right\} \\
& +b\left\|x_{n}-x_{n-1}\right\|+b\left\|y_{n}-y_{n-1}\right\|
\end{aligned}
$$

and:

$$
\begin{aligned}
& \left\|x_{n}-x_{n-1}\right\| \leq\left[\frac{a+c}{1-b}\right] \max \left\{\left\|x_{n-1}-x_{n-2}\right\|,\left\|y_{n-1}-y_{n-2}\right\|\right\} \\
& +\left[\frac{b}{1-b}\right]\left\|y_{n}-y_{n-1}\right\|
\end{aligned}
$$

Similarly:

$$
\begin{aligned}
& \left\|y_{n}-y_{n-1}\right\| \leq\left[\frac{a+c}{1-b}\right] \max \left\{\left\|x_{n-1}-x_{n-2}\right\|,\left\|y_{n-1}-y_{n-2}\right\|\right\} \\
& +\left[\frac{b}{1-b}\right]\left\|x_{n}-x_{n-1}\right\|
\end{aligned}
$$

Substituting from (2.9) into (2.8) gives: 


$$
\begin{aligned}
& \left\|x_{n}-x_{n-1}\right\| \leq\left[\frac{a+c}{1-b}\right] \max \left\{\left\|x_{n-1}-x_{n-2}\right\|, \| y_{n-1}-y_{n-2}\right\} \\
& +\left[\frac{b}{1-b}\right]\left[\frac{a+c}{1-b}\right] \max \left\{\left\|x_{n-1}-x_{n-2}\right\|,\left\|y_{n-1}-y_{n-2}\right\|\right\} \\
& +\left[\frac{b}{1-b}\right]\left[\frac{b}{1-b}\right]\left\|x_{n}-x_{n-1}\right\| \\
& \leq\left[\frac{a+c}{1-b}\right]\left[1+\frac{b}{1-b}\right] \max \left\{\left\|x_{n-1}-x_{n-2}\right\|,\left\|y_{n-1}-y_{n-2}\right\|\right\} \\
& \left.+\left[\frac{b}{1-b}\right]^{2}\left\|x_{n}-x_{n-1}\right\|\right]
\end{aligned}
$$

Hence:

$$
\begin{aligned}
& {\left[1-\left[\frac{b}{1-b}\right]^{2}\right]\left\|x_{n}-x_{n-1}\right\|} \\
& \leq\left[\frac{a+c}{1-b}\right]\left[1+\frac{b}{1-b}\right] \max \left\{\left\|x_{n-1}-x_{n-2}\right\|, \| y_{n-1}-y_{n-2}\right\}
\end{aligned}
$$

Hence:

$$
\begin{aligned}
& \left\|x_{n}-x_{n-1}\right\| \\
& \leq\left[\frac{a+c}{1-b}\right]\left[\frac{1+\frac{b}{1-b}}{1-\left[\frac{b}{1-b}\right]^{2}}\right] \max \left\{\left\|x_{n-1}-x_{n-2}\right\|,\left\|y_{n-1}-y_{n-2}\right\|\right\} \\
& \leq\left[\frac{a+c}{1-b}\right]\left[\frac{1-b}{1-2 b}\right] \max \left\{\left\|x_{n-1}-x_{n-2}\right\|,\left\|y_{n-1}-y_{n-2}\right\|\right\}
\end{aligned}
$$

Consequently:

$$
\left\|x_{n}-x_{n-1}\right\| \leq\left[\frac{a+c}{1-2 b}\right] \max \left\{\left\|x_{n-1}-x_{n-2}\right\|, \| y_{n-1}-y_{n-2}\right\}
$$

Similarly:

$$
\left\|y_{n}-y_{n-1}\right\| \leq\left[\frac{a+c}{1-2 b}\right] \max \left\{\left\|x_{n-1}-x_{n-2}\right\|, \| y_{n-1}-y_{n-2}\right\}
$$

Continuing $n-2$ same steps proves (2.6). The inequalities (2.7) can be proved by a similar way. Now, let $n, m \in N$ with $n \leq m$, we have:

$$
\begin{aligned}
& \left\|x_{m}-x_{n}\right\| \leq\left\|x_{m}-x_{m-1}\right\|+\ldots+\left\|x_{n-2}-x_{n-1}\right\|+\left\|x_{n-1}-x_{n}\right\| \\
& \leq\left[\left[\frac{a+c}{1-2 b}\right]^{m-1}+\ldots+\left[\frac{a+c}{1-2 b}\right]^{n-2}+\left[\frac{a+c}{1-2 b}\right]^{n-1}\right] K \\
& \leq \frac{\left[\frac{a+c}{1-2 b}\right]^{n}}{1-\left(\frac{a+c}{1-2 b}\right)} K
\end{aligned}
$$

Taking the limit as $n \rightarrow \infty$ proves that $\left\|x_{m}-x_{n}\right\| \rightarrow_{m, n \rightarrow \infty} 0$. because $\frac{a+c}{1-2 b}<1$.

Using the same steps of lemma (1) proves the following Lemma:

\section{Lemma 2}

Given a partially ordered normed space $X, T$ an $\{a, b, c\}$ second-type contraction mapping satisfying (2.3) and (2.4) and an upper-anti-lower couple point $\left(x_{0}, y_{0}\right)$ of $T$, then the inequalities (2.6) and (2.7) hold.

\section{Theorem 2}

Let $X$ be a sequentially lower-upper ordered Banach space $X$ and $T: X \times X \rightarrow X$ be a $\{a, b, c\} M$ first-type contraction mapping having the first-second mixed monotone and lower-upper properties on $X$. Then $T$ has first-anti-second couple fixed point in $X$. Moreover, for every lower-anti-upper couple $\left(x_{0}, y_{0}\right)$ of $T$ there is first-antisecond couple fixed point $(x, y)$ of $T$ such that the sequences (2.5) are converging strongly to $x$ and $y$ respectively, Also:

- If in addition $X$ is totally ordered and $(x, y)$ is a couple fixed of $T$, then $x=y$

- If in addition $X$ is totally ordered, $T$ on the diagonal satisfies $\|T(x, x)-T(y, y)\|<\|x-y\|$, then $T$ has a unique pair fixed, that is; there is $x \in X$ such that $T(x, x)=x$

\section{Proof}

Let $\left(x_{0}, y_{0}\right)$ be a lower-anti-upper couple of $T$. Then using lemma (1) shows that both $\left\{x_{n}\right\}_{n \in N}$ and $\left\{y_{n}\right\}_{n \in N}$ given in iteration (2.5) are Cauchy sequences in $X$, because of the completeness of $X$, these sequences are converging strongly to some elements $x$ and $y$ in $X$ respectively. Using the sequentially lower-upper ordered normed space assumption of $X$ gives $x_{n} \leq x, y \leq y_{n}$ for every $n \in N$, we get the following:

$$
\begin{aligned}
& \|T(x, y)-x\| \leq\left\|T(x, y)-x_{n}\right\|+\left\|x_{n}-x\right\| \\
& =\left\|T(x, y)-T\left(x_{n-1}, y_{n-1}\right)\right\|+\left\|x_{n}-x\right\| \\
& \leq a \max \left\{\left\|x-x_{n-1},\right\| y-y_{n-1} \|\right\} \\
& +b \max \{\|T(x, y)-x\|,\|T(y, x)-y\|\} \\
& +c \max \left\{\left\|T\left(x_{n-1}-y_{n-1}\right)-x_{n-1}\right\|,\left\|T\left(y_{n-1}, x_{n-1}\right)-y_{n-1}\right\|\right\} \\
& +\left\|x_{n}-x\right\|
\end{aligned}
$$

That is:

$$
\begin{aligned}
& \|T(x, y)-x\| \\
& \leq\left(\frac{1}{1-b}\right)\left[b\|T(y, x)-y\|+a \max \left\{\left\|x-x_{n-1}\right\|,\left\|y-y_{n-1}\right\|\right\}\right. \\
& \left.+c \max \left\{\left\|x_{n}-x_{n-1}\right\|,\left\|y_{n}-y_{n-1}\right\|\right\}+\left\|x_{n}-x\right\|\right]
\end{aligned}
$$


Taking the limit as $n \rightarrow \infty$ proves that:

$$
\|T(x, y)-x\| \leq\left(\frac{b}{1-b}\right)\|T(y, x)-y\|
$$

Similarly:

$$
\|T(y, x)-y\| \leq\left(\frac{b}{1-b}\right)\|T(x, y)-x\|
$$

thus:

$$
\begin{aligned}
& \|T(x, y)-x\| \leq\left(\frac{b}{1-b}\right)\left(\frac{b}{1-b}\right)\|T(y, x)-x\| \\
& =\left(\frac{b}{1-b}\right)^{2}\|T(x, y)-x\|
\end{aligned}
$$

Suppose that $\|T(x, y)-x\| \neq 0$, hence $1 \leq\left(\frac{b}{1-b}\right)^{2}, 1 \leq$ $\frac{b}{1-b}, 1-b \leq b, 1 \leq 2 b, b \geq \frac{1}{2}$ contradicting our assumption $b<\frac{1}{2}$, consequently, $\|T(x, y)-x\|=0$, hence $T(x, y)=x$, similarly $T(y, x)=y$ and $(x, y)$ is a couple fixed of $T$.

Now, given $X$ a totally ordered space, $(x, y)$ a couple fixed of $T$ and $x \neq y$. Then the following gives an obvious contradiction:

$$
\begin{aligned}
& \|x-y\|=\|T(x, y)-T(y, x)\| \\
& \leq a \max \{\|x-y\|,\|y-x\|\} \\
& +b \max \{\|T(x, y)-x\|,\|T(y, x)-y\|\} \\
& +c \max \{T(y, x)-y\|,\| T(x, y)-x \|\} \\
& =a\|x-y\|+(b+c) \max \{\|x-x\|,\|y-y\|\} \\
& =a\|x-y\|<\| x-y
\end{aligned}
$$

Finally, suppose that $X$ is totally ordered, $T$ on the diagonal satisfies $\|T(x, x)-\| T(y, y)\|<\| x-y \|$ and $(x, x)$ and $(y, y)$ are two pair fixed points with $x \neq y$. Then $\|x-y\|=$ $\|T(x, x)-\| T(y, y)\|<\| x-y \|$ is a contradiction.

\section{Corollary 2}

Let $X$ be a sequentially lower-upper ordered complete normed space and $T: X \times X \rightarrow X$ be a $\{a, b, c\}$ first-type contraction mapping having the first-second mixed monotone and lower-upper properties on $X$. Then $T$ has a first-anti-second couple fixed point in $X$. Moreover, for every lower-anti-upper couple $\left(x_{0}, y_{0}\right)$ of $T$ there is first-anti-second couple fixed point $(x, y)$ of $T$ such that the sequences (2.5) are converging strongly to $x$ and $y$ respectively. Also:
- If in addition $X$ is totally ordered and $(x, y)$ is a couple fixed of $T$, then $x=y$

- If in addition $X$ is totally ordered, $T$ on the diagonal satisfies $\|T(x, x)-\| T(y, y)\|<\| x-y \|$, then $T$ has a unique fixed point

\section{Proof}

Using:

$$
\begin{aligned}
& \|T(x, y)-T(u \cdot v)\| \\
& \leq a \max \{\|x-u\|,\|y-v\|\}+b\|T(x, y)-x\| \\
& +c \max \{\|T(u, v)-u\|,\|T(v, u)-v\|\} \\
& \leq a \max \{\|x-u\|,\|y-v\|\} \\
& +b \max \{\|T(x, y)-x\|,\|T(y, x)-y\|\} \\
& +c \max \{\|T(u, v)-u\|,\|T(v, u)-v\|\}
\end{aligned}
$$

for all $x, y, u$ and $v$ in $X$ with $x \geq u$ and $y \leq v$ shows that every $\{a, b, c\}$ first-type contraction mapping is $\{a, b, c\}$ $\mathrm{M}$ first-type contraction mapping, using Theorem (2) completes the proof.

\section{Conclusion}

We introduced new class for contraction types of mappings, the mappings defined from normed space into its Cartesian product, proved that this defined class is lying between the nonexpansive and quasi nonexpansive classes and studied the concept of fixed points for the mappings in this new defined class to extend the concept of $C$ contractivity condition studied in (Suzuki, 2008). We also defined new contraction types of mappings with a mixed monotone property, $M$ first and $M$ second contraction types of mappings, the mappings defined from the Cartesian product of a sequentially ordered Banach space into itself and proved the existence of pair, first-anti-second and second-anti-first couple fixed points of such mappings to generalize some of the results given in (Ali, 2013).

\section{Acknowledgement}

Sincere thanks go to the reviewer valuable comments that strength the value of this research paper.

\section{Ethics}

The author declares that there is no conflict of interest in the paper.

\section{References}

Ali, S.M., 2013. Fixed point theorems of some type contraction mappings. J. Nonlinear Convex Anal., 14: 331-342. 
Browder, F.E., 1965. Nonexpansive nonlinear operators in a banach space. Proc. Natl. Acad. Sci. USA, 54: 1041-1044. DOI: 10.1073/pnas.54.4.1041

Gnana Bhaskar, T. and V. Lakshmikantham, 2006. Fixed point theorems in partially ordered metric spaces and applications. Nonlinear Anal., 65: 1379-1393. DOI: 10.1016/j.na.2005.10.017

Jain, M., K. Tas, B.E. Rhoades and N. Gupta, 2014. Coupled fixed point theorems for generalized symmetric contractions in partially ordered metric spaces and applications. J. Comput. Anal. Applic.
Suzuki, T., 2008. Fixed point theorems and convergence theorems for some generalized nonexpansive mappings. J. Math. Anal. Applic., 340: 1088-1095. DOI: 10.1016/j.jmaa.2007.09.023

Turkoglu, D., 2011. Some fixed-point theorems for multivalued monotone mappings in ordered uniform space. Fixed Point Theory Applic., 2011: 186237-186237. DOI: 10.1155/2011/186237 